Research Journal of Applied Sciences, Engineering and Technology 6(19): 3559-3567, 2013

DOI:10.19026/rjaset.6.3560

ISSN: 2040-7459; e-ISSN: 2040-7467

(C) 2013 Maxwell Scientific Publication Corp.

Submitted: December 17, $2012 \quad$ Accepted: January 19, $2013 \quad$ Published: October 20, 2013

\title{
Research Article \\ Robust Output Feedback Control for Active Seat Suspension Systems with Actuator Time Delay Using $\mu$-Synthesis Approach
}

\author{
${ }^{1}$ Mohammad Gudarzi, ${ }^{2}$ Atta Oveisi and ${ }^{2}$ Mohammad Mahdi Mohammadi \\ ${ }^{1}$ School of Mechanical Engineering, Damavand Branch, Islamic Azad University, Tehran, Iran \\ ${ }^{2}$ Department of Mechanical Engineering, Iran University of Science and Technology, Tehran, Iran
}

\begin{abstract}
This study presents a robust output feedback optimal $H_{\infty}$ control synthesis for a class of uncertain seat suspension systems with actuator saturation and an uncertain actuator time delay. A vertical vibration model of human body is added in order to make the modeling of seat suspension systems more accurate. A dynamic controller is considered by using of two measurable states of the model, by real sensors, as output feedback. Moreover, uncertain actuator time delay is considered to guarantee robust performance of the closed-loop system. The controller is derived by using D-K iteration algorithm for constrained systems with norm-bounded uncertainties. The corresponding closed-loop system is asymptotically stable with a guaranteed $\mathrm{H}_{\infty}$ performance. Finally, a design example is presented to show the performance and robustness of the developed theoretical results.
\end{abstract}

Keywords: Active suspension, D-K iteration, output feedback, robust performance, uncertain time delay

\section{INTRODUCTION}

Vehicle suspensions have been a hot research topic for many years due to its important role in ride comfort, vehicle safety, road damage minimization and the overall vehicle performance (Gao et al., 2006). There are three main types of vehicle suspensions that have been proposed, that is, passive, semi-active and active suspensions, which depend on the operation mode to achieving the mentioned goals (Du et al., 2005). Conventional passive suspensions are effective only in a certain frequency range and optimal design performance cannot be achieved when the system and its operating conditions are changed. On the contrary, it has been well recognized that active suspensions have a great potential to meet the tight performance requirements demanded by users. Therefore, in recent years more and more attention has been devoted to the development of active suspensions and various approaches have been proposed to solve the crucial problem of designing a suitable control law for these active suspension systems (Yoshimura et al., 1986; Watanabe and Sharp, 1999; Roh and Park, 1999; Chou et al., 1998; Salman et al., 1990; El Madany and AlMajed, 2001; Guo and Zhang, 2012).

In addition to aforementioned objects, another important goal of controller design is to maintain the robustness of the closed-loop systems (Yamashita et al., 1994). In linear control theories, plants are assumed to be linear and time-invariant systems. However, in real vehicles the total vehicle mass varies due to changes in passenger load and cargo and characteristics of the actuators change due to aging and nonlinearities. Thus, real plants are time-variant systems. When parameters in the plants change like this, the control performance specified in the design stage tends to deteriorate. However, a constant performance is desired in automotive suspensions, so, this deterioration has to be kept as small as possible. Therefore, the analysis and synthesis of robust control for active suspension systems has become a research concern (Ray, 1991; Kiriczi and Kashani, 1991; Ma et al., 2012).

In active control of vehicle suspension systems, the time delay of the system is another important issue that needs careful treatment to avoid poor performance or even possible instability of the closed-loop system. On the other hand, time delays are widely encountered in these kinds of control loops because of the electrical and electromagnetic characteristics of the actuators. As a result, they have been widely studied during the past decades and many analytical techniques and synthesis methods have been developed using delay-dependent Lyapunov function concerning conservatism (Gao and Wang, 2003; He et al., 2004; Park, 1999; Shi et al., 1999; Wang et al., 2005, 2006; Wu et al., 2006).

The most previous researches concerning with seat suspensions have limited their scope to model the diver as a rigid dummy mass on the seat, which is obviously not precise enough to investigate the performances because no bio-dynamics are included (Li et al., 2012; $\mathrm{Du}$ and Zhang, 2007). Therefore, the sophisticated research of ride comfort and safety improvement calls

Corresponding Author: Mohammad Gudarzi, School of Mechanical Engineering, Damavand Branch, Islamic Azad University, Tehran, Iran

This work is licensed under a Creative Commons Attribution 4.0 International License (URL: http://creativecommons.org/licenses/by/4.0/) 
for a mathematical seated human body model. In addition, some researchers have used state-feedback approach (Esmailzadeh and Taghirad, 1998; Meng et al., 2009; Zhao et al., 2010), while this method is not applicable, because of the limitations in sensors attachment especially in bio-dynamics part of the model, in real systems.

In this study, the problem of robust optimal $\mathrm{H}_{\infty}$ output feedback controller design for a class of active seat suspension systems with uncertain actuator input time delay, norm-bounded parameter uncertainties and actuator saturation is investigated. In order to obtain a better insight of the suspension system performance, a vibration model of human body is introduced and combined with the seat. The robust controller design method is also considered in this study to ensure the closed-loop system asymptotical stability and $\mathrm{H}_{\infty}$ performance in spite of the parameter uncertainties. Actuator saturation is considered in the procedure of control design by adding the actuator force to the augmented plant desired output vector. In addition, an uncertain actuator time delay is considered in the augmented plant control input. From six states of the seat suspension model, just two measurable, by real sensors, states are considered as system output feedback. Finally, desired dynamic controller has been obtained using D-K iteration procedure, then the responses of the closed-loop system with some different actuator time delays has been compared with the open loop one and results have been discussed.

\section{DYNAMIC MODELING}

In this study, a three-degree-of-freedom seat suspension model shown in Fig. 1 established by Wei and Griffin (1998) is considered for controller design.

In this figure, $m_{1}$ is the mass of seat frame; $m_{21}$ and $m_{22}$ are the masses of human thighs together with buttocks and these at cushion, respectively and $m_{2}=m_{21}+m_{22} ; m_{3}$ is the mass of the upper body of a seated human. The mass of lower legs and feet is neglected because of their little contribution to the biodynamic response of the seated body. $c_{1}, c_{2}$ and $k_{1}$, $k_{2}$ are dampings and stiffnesses of the passive suspension system, respectively; $c_{3}$ and $k_{3}$ stand for the damping and stiffness of the components inside human body such as spines; $z_{1}, z_{2}$ and $z_{3}$ are the displacements of the corresponding masses; $z_{0}$ is the road displacement input, $\omega(t)=\dot{z}_{0}(t)$ represents the disturbance caused by road roughness; $u$ is the active control input of the seat suspension system. To predict the biodynamic responses more reasonably, the mass of buttocks and legs is assumed to contact rigidly with the seat. The road excitation input is transmitted to the cabin floor. It is also assumed that only the vertical motion of the vehicle exists for simplification. The governing equations of motion for the seat suspension can be expressed as:

$$
m_{1} \ddot{z}_{1}=-c_{1}\left(\dot{z}_{1}-\dot{z}_{0}\right)-k_{1}\left(z_{1}-z_{0}\right)+c_{2}\left(\dot{z}_{2}-\dot{z}_{1}\right)
$$

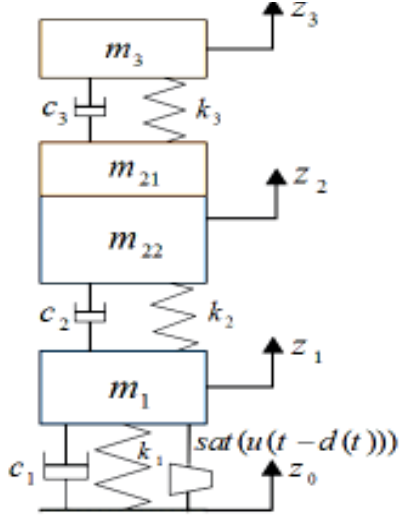

Fig. 1: Dynamic model of the seat suspension system

$$
\begin{aligned}
& +k_{2}\left(z_{2}-z_{1}\right)-u \\
& \begin{array}{c}
m_{2} \ddot{z}_{2}=-c_{2}\left(\dot{z}_{2}-\dot{z}_{1}\right)-k_{2}\left(z_{2}-z_{1}\right)+c_{3}\left(\dot{z}_{3}-\dot{z}_{2}\right) \\
\quad+k_{3}\left(z_{3}-z_{2}\right)
\end{array} \\
& m_{3} \ddot{z}_{3}=-c_{3}\left(\dot{z}_{3}-\dot{z}_{2}\right)-k_{3}\left(z_{3}-z_{2}\right)
\end{aligned}
$$

By defining the state variable as:

$$
\begin{aligned}
& x(t)= \\
& {\left[\begin{array}{llllll}
x_{1}(t) & x_{2}(t) & x_{3}(t) & x_{4}(t) & x_{5}(t) & x_{6}(t)
\end{array}\right]^{T}(2)}
\end{aligned}
$$

where,

$$
\begin{aligned}
& x_{1}(t)=z_{1}(t)-z_{0}(t) \\
& x_{2}(t)=\dot{z}_{1}(t) \\
& x_{3}(t)=z_{2}(t)-z_{1}(t) \\
& x_{4}(t)=\dot{z}_{2}(t) \\
& x_{5}(t)=z_{3}(t)-z_{2}(t) \\
& x_{6}(t)=\dot{z}_{3}(t)
\end{aligned}
$$

which are the deflections of the corresponding springs and velocities of the mass segments. Then the dynamic Eq. (1) can be written in the following state-space form:

$$
\dot{x}(t)=A x(t)+B u(t)+B_{w} \omega(t)
$$

where,

$$
\begin{gathered}
A=\left[\begin{array}{cccccc}
0 & 1 & 0 & 0 & 0 & 0 \\
-\frac{k_{1}}{m_{1}} & -\frac{c_{1}+c_{2}}{m_{1}} & \frac{k_{2}}{m_{1}} & \frac{c_{2}}{m_{1}} & 0 & 0 \\
0 & -1 & 0 & 1 & 0 & 0 \\
0 & \frac{c_{2}}{m_{2}} & -\frac{k_{2}}{m_{2}} & -\frac{c_{2}+c_{3}}{m_{2}} & \frac{k_{3}}{m_{2}} & \frac{c_{3}}{m_{2}} \\
0 & 0 & 0 & -1 & 0 & 1 \\
0 & 0 & 0 & \frac{c_{3}}{m_{3}} & -\frac{k_{3}}{m_{3}} & -\frac{c_{3}}{m_{3}}
\end{array}\right] \\
B=\left[\begin{array}{llllll}
0 & -\frac{1}{m_{1}} & 0 & 0 & 0 & 0
\end{array}\right]^{T}
\end{gathered}
$$




$$
B_{w}=\left[\begin{array}{llllll}
-1 & -\frac{c_{1}}{m_{1}} & 0 & 0 & 0 & 0
\end{array}\right]^{T}
$$

The seat suspension model becomes an uncertain model when changes in vehicle inertial properties, actuator time delays and saturation nonlinearities are taken into account, which can be expressed as:

$$
\dot{x}(t)=\bar{A} x(t)+\bar{B} \sigma(u(t-d(t)))+B_{w} w(t)
$$

The uncertain actuator delay $d$ is an unknown magnitude that satisfies:

$$
d_{1} \leq d \leq d_{2}
$$

where $d_{1}$ and $d_{2}$ are the lower and upper bounds of the control input delay, respectively. Mentioned time delay can be modeled as a $e^{-d s}$ gain in the control system, or can be estimated as a rational form:

$$
e^{-d s} \approx \frac{-\frac{d}{2} s+1}{\frac{d}{2} s+1}
$$

The parameter uncertainties are considered as norm-bounded uncertainties with the form:

$$
\begin{aligned}
& \bar{A}=A+\Delta A, \bar{B}=B+\Delta B \\
& {\left[\begin{array}{ll}
\Delta A & \Delta B
\end{array}\right]=L_{1} F(t)\left[\begin{array}{ll}
E_{A} & E_{B}
\end{array}\right]}
\end{aligned}
$$

where $L_{1}, E_{A}, E_{B}$ are known constant real matrices of appropriate dimensions and $F(t)$ is an unknown matrix function with Lebesgue-measurable elements satisfying $F^{T}(t) F(t) \leq I$ and the actuator saturation nonlinearity is described by:

$$
\begin{aligned}
& \sigma(u(t))=\left[\begin{array}{llll}
\sigma\left(u_{1}(t)\right) & \sigma\left(u_{2}(t)\right) & \ldots & \sigma\left(u_{q}(t)\right)
\end{array}\right]^{T} \\
& \sigma\left(u_{i}(t)\right) \triangleq \\
& \left\{\begin{array}{c}
u_{i \max } \text { if } u_{i}(t) \geq u_{i \max } \\
u_{i}(t) \text { if }-u_{i \max } \leq u_{i}(t) \leq u_{i \max } \\
-u_{i \max } \text { if } u_{i}(t) \leq-u_{i \max }
\end{array}\right.
\end{aligned}
$$

Before designing the state feedback control law for a seat suspension system, we need to consider the following aspects:

Ride comfort: Ride comfort can be generally quantified by the body acceleration in the vertical direction, thus, it is chosen as the first control output, i.e., minimizing the vertical acceleration of human body $\ddot{z}_{3}(t)$ is one of our most concerned objectives in the controller design, that is:

$$
z_{o 1}(t)=\ddot{z}_{3}(t)
$$

Moreover, the $H_{\infty}$ norm is employed to measure the performance, whose value actually gives an upper bound of the root-mean-square gain. Hence, our goal is to minimize the $H_{\infty}$ norm of the transfer function from the disturbance $w(t)$ to the control output $z_{01}(t)$ in order to improve ride comfort.

Actuator saturation: The maximum value of the actuator force is limited due to its physical properties. So, the controller should be designed by considering this limitation to obtain an appropriate performance:

$$
z_{o 2}(t)=u(t) \leq u_{\max }
$$

Suspension deflection limitation: The controller should be capable to prevent the suspension from hitting its travel limit in order to avoid ride comfort deterioration and mechanical structural damage. The requirement is:

$$
z_{o 3}(t)=\left|z_{1}(t)-z_{0}(t)\right| \leq z_{\max }
$$

where $z_{\max }$ is the maximum suspension deflection limit, under all road disturbance inputs. The deflection space does not need to be minimized but its peak value needs to be limited.

Therefore, the strategy in the seat suspension system control law designing is to minimize the $H_{\infty}$ norm of the closed-loop transfer function from the disturbance $w(t)$ to the weighted control output $Q z_{o 1}(t)+R z_{o 2}(t)$ and guarantee the suspension stroke requirement. Note that $Q$ and $R$ are two weighting coefficients.

In a real suspension, all six states are not measurable. $x_{1}$ and $x_{3}$ are two measurable states in practice using appropriate sensors (note that $x_{5}$ and $x_{6}$ because of their relation to human body movement, are immeasurable in a real suspension system.), so the system outputs that are using in the feedback control law will be as bellow:

$$
y(t)=\left[\begin{array}{ll}
x_{1}(t) & x_{3}(t)
\end{array}\right]^{T}
$$

Then, the vehicle seat suspension system can be described by the following state-spaces equations:

$$
\begin{aligned}
& \dot{x}(t)=\bar{A} x(t)+\bar{B} \sigma(u(t-d(t)))+B_{w} w(t) \\
& z_{o 1}(t)=\bar{C}_{1} x(t) \\
& z_{o 2}(t)=u(t) \\
& z_{o 3}(t)=\bar{C}_{2} x(t) \\
& y(t)=\bar{C}_{3} x(t)
\end{aligned}
$$

where $\bar{A}, \bar{B}, B_{w}$ are already defined in (5) and:

$$
\begin{aligned}
& \bar{C}_{1}=\left[\begin{array}{llllll}
0 & 0 & 0 & \frac{c_{3}}{m_{3}} & -\frac{k_{3}}{m_{3}} & -\frac{c_{3}}{m_{3}}
\end{array}\right] \\
& \bar{C}_{2}=\left[\begin{array}{llllll}
1 & 0 & 0 & 0 & 0 & 0
\end{array}\right]
\end{aligned}
$$


Res. J. Appl. Sci. Eng. Technol., 6(19): 3559-3567, 2013

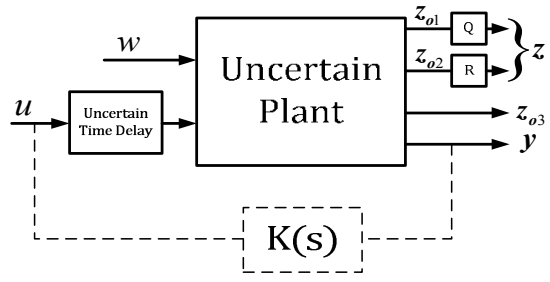

Fig. 2: Augmented plant for the modeled suspension

$$
\bar{C}_{3}=\left[\begin{array}{llllll}
1 & 0 & 0 & 0 & 0 & 0 \\
0 & 0 & 1 & 0 & 0 & 0
\end{array}\right]
$$

With $\bar{C}_{1}=C_{1}+\Delta C_{1}, \Delta C_{1}=L_{2} F(t) E_{c_{1}}$ and $\bar{C}_{3}=C_{3}+$ $\Delta C_{3}, \Delta C_{3}=L_{3} F(t) E_{c_{3}}$. In this study, our goal is to find an output-feedback control system:

$$
\begin{aligned}
& \dot{\zeta}(t)=A_{c} \varsigma(t)+B_{c} y(t) \\
& u(t)=C_{c} \varsigma(t)+D_{c} y(t)
\end{aligned}
$$

Such that the following requirements are satisfied:

- The closed-loop system is asymptotically stable.

- Under zero initial condition, the performance $\left\|T_{z_{01} w}\right\|_{\infty}<\gamma$ is minimized subject to (10) for all nonzero $w \in L_{2}[0, \infty)$, where $\left.T_{\left[Q z_{01}\right.} R z_{o 2}\right]^{T} w$ denotes the closed-loop transfer function from the road disturbance $w(t)$ to the weighted control output $Q z_{o 1}(t)+R z_{o 2}(t)$.

Finally, the augmented plant by considering mentioned objectives, input, outputs and time delay for controller designing will be as Fig. 2.

\section{CONTROLLER DESIGN}

In order to apply the general structured singular value theory to control system design, the control problem should be recast into the Linear Fractional Transformation (LFT) setting as in Fig. 3.

In the Fig. 3, $P(s)$ is the open-loop interconnection and contains all of the known elements including the nominal plant model, performance and uncertainty weighting functions. The $\Delta_{\text {pert }}$ block is the uncertain element from the set $\Delta_{\text {pert }}$, which parameterizes all of the assumed model uncertainty in the problem and $K(s)$ is the controller. Three sets of inputs consist of perturbation $w$, disturbances $d$ and controls $u$ enter $P(s)$. And three sets of outputs consist of perturbation outputs $z$, errors $e$ and measurements $y$ are generated.

The set of systems to be controlled is described by the LFT as:

$\left\{F_{U}\left(P(s), \Delta_{\text {pert }}\right): \Delta_{\text {pert }} \in\right.$ $\Delta$ pert, $\operatorname{MAX} \Omega \sigma \Delta \operatorname{pert}(j \omega) \leq 1$

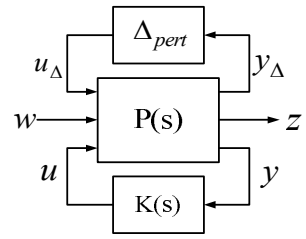

Fig. 3: LFT description of control problem

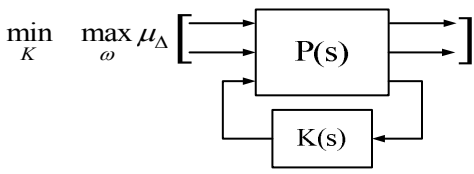

Fig. 4: $\mu$-synthesis concept

The design objective is to find a stabilizing controller $K(s)$, such that for all such perturbations $\Delta_{\text {pert }}$, the closed-loop system is stable and satisfies:

$$
\left\|F_{L}\left[F_{U}\left(P(s), \Delta_{\text {pert }}\right), K(s)\right]\right\|_{\infty}<1
$$

But,

$$
\begin{aligned}
& F_{L}\left[F_{U}\left(P(s), \Delta_{\text {pert }}\right), K(s)\right] \\
& =F_{U}\left[F_{L}(P(s), K(s)), \Delta_{\text {pert }}\right]
\end{aligned}
$$

Therefore, the design objective is to find a nominally stabilizing controller $K(s)$, such that for all $\Delta_{\text {pert }} \in \Delta_{\text {pert }}, \max _{\omega} \bar{\sigma}\left[\Delta_{\text {pert }}(j \omega)\right] \leq 1$ the closed-loop system is stable and satisfies:

$$
\left\|F_{U}\left[F_{L}(P(s), K(s)), \Delta_{\text {pert }}\right]\right\|_{\infty}<1
$$

Given any $K(s)$, this performance objective can be checked utilizing a robust performance test on the linear fractional transformation $F_{L}(P(s), K(s))$. The robust performance test should be computed with respect to an augmented uncertainty structure. The structured singular value provides the correct test for robust performance. $K(s)$ achieves robust performance if and only if:

$$
\max _{\omega} \mu_{\Delta}\left(F_{L}(P(s), K(s))(j \omega)\right)<1
$$

The goal of $\mu$-synthesis is to minimize over all stabilizing controllers $K(s)$, the peak value of $\mu_{\Delta}($.$) of$ the closed-loop transfer function $F_{L}(P(s), K(s))$. More formally:

$\min \underset{\text { stabilizing }}{K} \max _{\omega} \mu_{\Delta}\left(F_{L}(P(s), K(s))(j \omega)\right)<1$

This aim is shown in Fig. 4. For tractability of the $\mu$ synthesis problem it is necessary to replace $\mu_{\Delta}($. with the upper bound. For a constant matrix $M$ and an uncertainty structure $\Delta$, an upper bound for $\mu_{\Delta}(M)$ is an optimally scaled maximum singular value: 


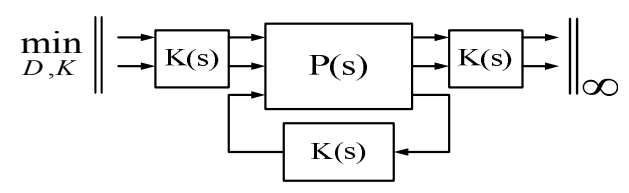

Fig. 5: Replacing $\mu$ with upper bound

$$
\mu_{\Delta}(M) \leq \inf _{D \in \mathbf{D}_{\Delta}} \bar{\sigma}\left(D M D^{-1}\right)
$$

where $D_{\Delta}$ is the set of matrices with the property that $D \Delta=\Delta D$ for every $D \in D_{\Delta}, \Delta \in \Delta$. Using this upper bound, the optimization in equation is reformulated as:

$$
\min \underset{\text { stabilizing }}{K} \max _{\omega} \min _{D_{\omega} \in \mathbf{D}_{\Delta}} \bar{\sigma}\left[\begin{array}{c}
D_{\omega} F_{L}(P(s), K(s)) \\
(j \omega) D_{\omega}^{-1}
\end{array}\right]
$$

The $D$ minimization is simply an approximation to $\left[F_{L}(P(s), K(s))(j \omega)\right] . D_{\omega}$ is chosen from the set of scalings, $\mathrm{D}_{\Delta}$, independently at every $\omega$. So:

$$
\min _{K} \min _{\text {stabilizing }} \min _{D, D_{\omega} \in \mathrm{D}_{\Delta}} \max _{\omega} \bar{\sigma}\left[\begin{array}{c}
D_{\omega} F_{L}(P(s), K(s)) \\
(j \omega) D_{\omega}^{-1}
\end{array}\right]
$$

$D_{,} D_{\omega} \in \mathrm{D}_{\Delta}, \quad$ means a frequency-dependent function $D$ that satisfies $D_{\omega} \in \mathrm{D}_{\Delta}$ for each $\omega$. The general expression $\max _{\omega} \bar{\sigma}[f(\omega)]$ is noted as $\|f\|_{\infty}$, giving:

$$
\min \underset{\text { stabilizing }}{K} \min _{D, D_{\omega} \in \mathrm{D}_{\Delta}}\left\|D F_{L}(P, K) D^{-1}\right\|_{\infty}
$$

Consider a single matrix $D \in \mathrm{D}_{\Delta}$ and a complex matrix $M$. Suppose that $U$ is a complex matrix with the same structure as $D$, but satisfying $U^{*} U=U U^{*}=I$. Each block of $U$ is a unitary (orthogonal) matrix. Matrix multiplication by an orthogonal matrix does not affect the maximum singular value, hence:

$$
\begin{aligned}
& \bar{\sigma}\left[(U D) M(U D)^{-1}\right]=\bar{\sigma}\left[U D M D^{-1} U^{*}\right] \\
& =\bar{\sigma}\left[D M D^{-1}\right]
\end{aligned}
$$

Therefore, replacing $D$ by $U D$ does not affect the upper bound. Using this freedom in the phase of each block of $D$, the frequency-dependent scaling matrix $D_{\omega}$ can be restricted to be a real-rational, stable, minimumphase transfer function, $\widehat{D}(s)$ and not affect the value of the minimum. Hence the new optimization is:

$$
\min \underset{\text { stabilizing }}{K} \min \underset{\text { stable,min-phase }}{\widehat{D}(s) \in \mathbf{D}_{\Delta}}\left\|\widehat{D} F_{L}(P, K) \widehat{D}^{-1}\right\|_{\infty}
$$

This optimization is currently solved by an iterative approach, referred to as $D-K$ iteration. A block diagram depicting the optimization is shown in Fig. 5.

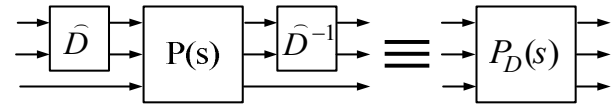

Fig. 6: Replacing rational $D$ scaling

To solve optimization problem, in the first stage consider holding $D(s)$ fixed at a given, stable, minimum phase, real-rational $\widehat{D}(s)$. Then, solve the optimization:

$$
\underset{\text { stabilizing }}{K}\left\|\widehat{D} F_{L}(P, K) \widehat{D}^{-1}\right\|_{\infty}
$$

Define $P_{D}$ to be the system shown in Fig. 6. So, the optimization is equivalent to:

$$
\min \underset{\text { stabilizing }}{K}\left\|F_{L}\left(P_{D}, K\right)\right\|_{\infty}
$$

Since $P_{D}$ is known at this step, this optimization is precisely an $H_{\infty}$ optimization control problem. The solution to the $H_{\infty}$ problem is well known and involves solving algebraic Riccati equations in terms of a statespace model for $P_{D}$.

In the second stage with $K$ held fixed, the optimization over $D$ is carried out in a two-step procedure:

- Finding the optimal frequency-dependent scaling matrix $D$ at a large, but finite set of frequencies (this is the upper bound calculation for $\mu$ ).

- Fitting this optimal frequency-dependent scaling with a stable, minimum-phase, real-rational transfer function $\widehat{D}$.

The two-step procedure is a viable and reliable approach. The primary reason for its success is the efficiency with which both of the individual steps are carried out.

\section{NUMERICAL RESULTS AND DISCUSSION}

In order to evaluate the effectiveness and robust performance of the controller design method proposed in the above section, an example has been introduced in this section. The schematic and biodynamical parameters for this study are listed in Table 1 (Liang and Chiang, 2006; Choi and Han, 2007; Zhao et al., 2010). The maximum suspension deflection is defined as $z_{\text {max }}=0.08 \mathrm{~m}$, the maximum control force is assumed as $u_{\max }=1500 \mathrm{~N}$. Furthermore, assume that the input time delay lower bound $d_{\min }=0 \mathrm{~ms}$, upper bound $d_{\max }=25 \mathrm{~ms}$ and the norm-bounded parameter uncertainties are expressed as: 
Res. J. Appl. Sci. Eng. Technol., 6(19): 3559-3567, 2013

Table 1: System parameters of the modeled seat suspension

\begin{tabular}{|c|c|c|c|c|c|}
\hline \multicolumn{2}{|c|}{ Mass $(k g)$} & \multicolumn{2}{|c|}{ Damping coefficient $(\mathrm{N} \mathrm{s} / \mathrm{m})$} & \multicolumn{2}{|c|}{ Spring constant $(N / m)$} \\
\hline Symbol & Magnitude & Symbol & Magnitude & Symbol & Magnitude \\
\hline$m_{1}$ & 15 & $c_{1}$ & 830 & $k_{1}$ & 31000 \\
\hline$m_{2}$ & $1+7.8$ & $c_{2}$ & 200 & $k_{2}$ & 18000 \\
\hline$m_{3}$ & 43.3 & $c_{3}$ & 1485 & $k_{3}$ & 44130 \\
\hline
\end{tabular}
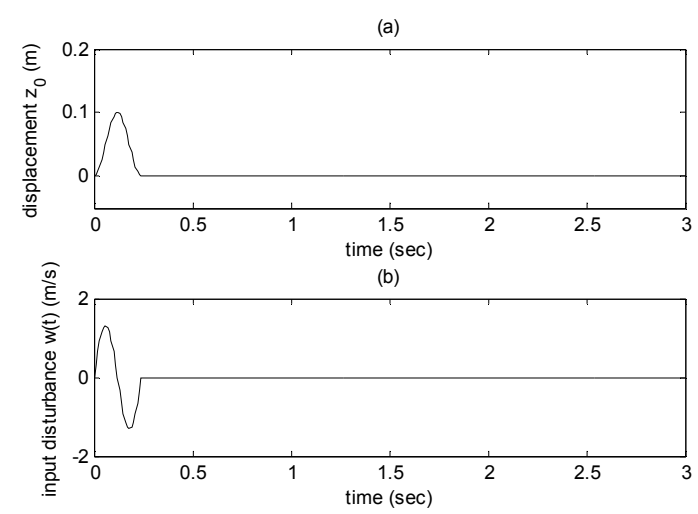

Fig. 7: Bump displacement (a) and input disturbance (b) from ground

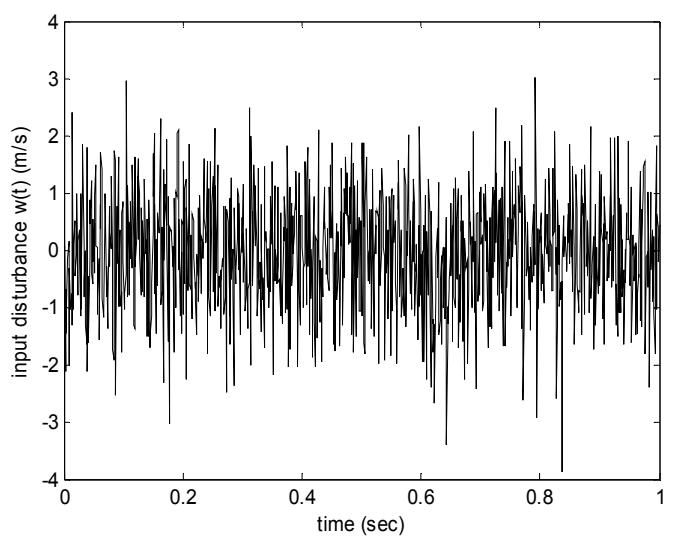

Fig. 8: Zero-mean white noise input disturbance from ground

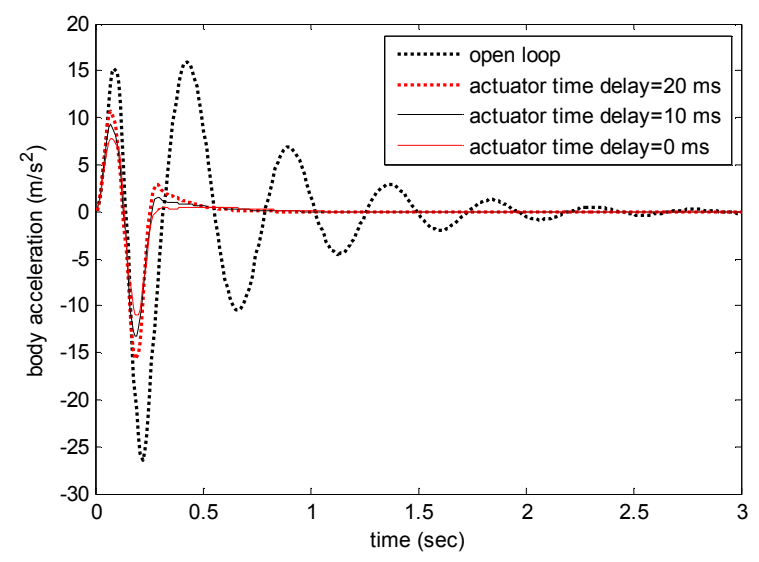

Fig. 9: Vertical accelerations of open-loop system and closedloop system with some actuator time delays under bump excitation

$$
\begin{aligned}
& L_{1}=\delta_{1} \times\left[\begin{array}{llllll}
0 & 0 & 0 & 0 & 0 & 0 \\
0 & 0 & 0 & 0 & 0 & 0 \\
0 & 0 & 0 & 0 & 0 & 0 \\
0 & 1 & 1 & 1 & 1 & 1 \\
0 & 0 & 0 & 0 & 0 & 0 \\
0 & 0 & 0 & 1 & 1 & 1
\end{array}\right] \\
& L_{2}=\delta_{2} \times\left[\begin{array}{llllll}
0 & 0 & 0 & 1 & 1 & 1
\end{array}\right] \\
& L_{3}=\delta_{3} \times\left[\begin{array}{llllll}
1 & 0 & 0 & 0 & 0 & 0 \\
0 & 0 & 1 & 0 & 0 & 0
\end{array}\right] \\
& E_{B}=\delta_{B} \times\left[\begin{array}{l}
0 \\
1 \\
0 \\
0 \\
0 \\
0
\end{array}\right]
\end{aligned}
$$

$E_{A}=\delta_{A} I, \quad E_{C_{1}}=\delta_{C_{1}} I, \quad E_{C_{3}}=\delta_{C_{3}} I \quad$ where $\delta_{1}, \delta_{2}$, $\delta_{3}, \delta_{A}, \delta_{B}, \delta_{C_{1}}, \delta_{C_{3}}$ are all set to 0.02 for simplicity.

For investigating of the seat suspension performance, road disturbances can be generally assumed as shocks. Shocks are discrete events of relatively short duration and high intensity, caused by, for example, a pronounced bump or pothole on an otherwise smooth road surface. In this study, this case of road profile is considered first to reveal the transient response characteristic, which is given by:

$$
Z_{0}(t)=\left\{\begin{array}{cc}
\frac{a}{2}\left(1-\cos \left(\frac{2 \pi V_{0}}{l} t\right)\right), & 0 \leq t \leq \frac{l}{V_{0}} \\
0, & t>\frac{l}{V_{0}}
\end{array}\right.
$$

and illustrated in Fig. 7a, where $a$ is the height of the bump and $l$ is the length of the bump. Here we choose $a=0.1 \mathrm{~m}, l=2 \mathrm{~m}$ and the vehicle forward velocity $V_{0}=30(\mathrm{~km} / \mathrm{h})$. Note that the input disturbance to the system is as Fig. 7b. The second type of input disturbance from the ground $w(t)$ is assumed to be zero-mean white noise with identity power spectral density, which is shown in Fig. 8.

The bump responses of the passive suspension and the active suspension with designed controller for nominal system and with some different actuator time delays compared in Fig. 9. It demonstrates that the closed-loop system is asymptotically stable and has a better performance with or without actuator time delays. However, the closed-loop performance degrades significantly when the actuator delay gets larger.

The bump response of suspension deflection is plotted in Fig. 10, from which it can be seen that this 


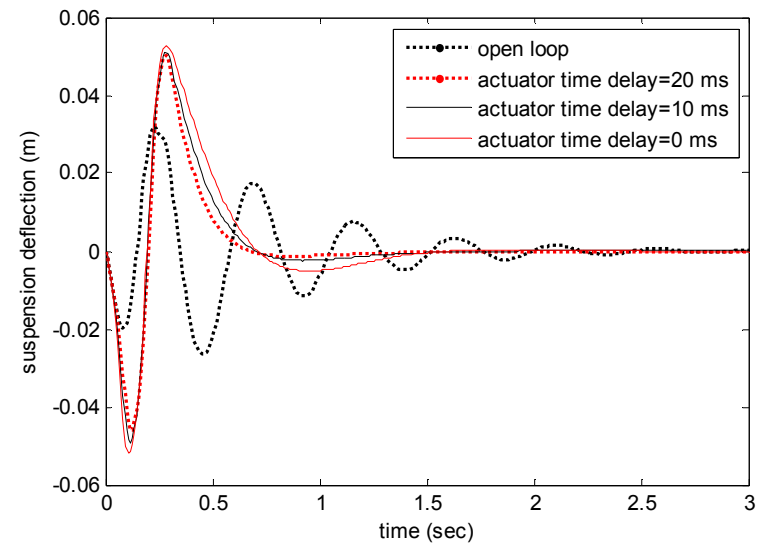

Fig. 10: Suspension deflections of open-loop system and closed-loop system with some actuator time delays under bump excitation

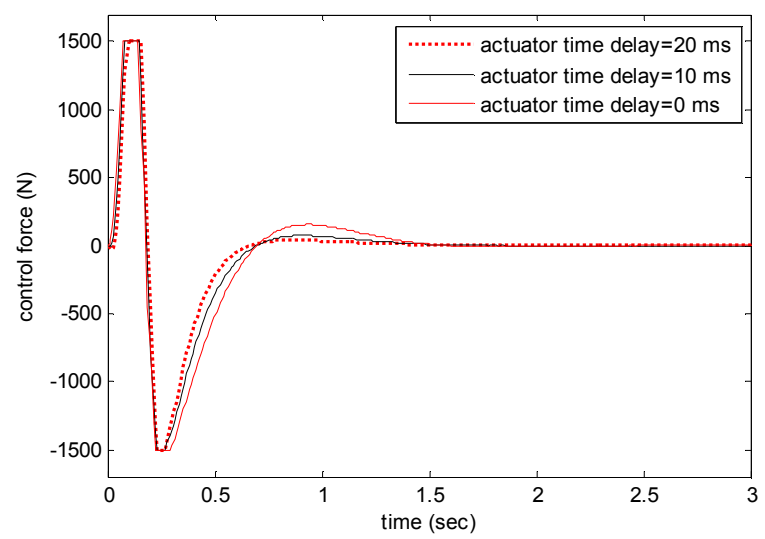

Fig. 11: Control forces of closed-loop system with some actuator time delays under bump excitation

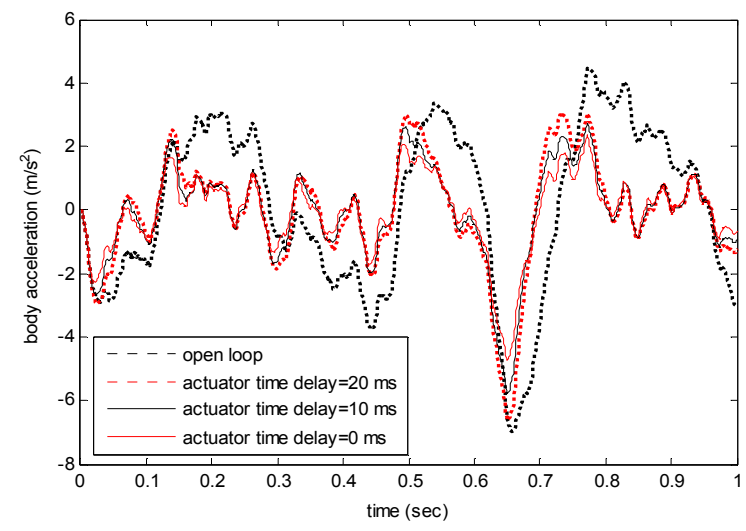

Fig. 12: Vertical accelerations of open-loop system and closed-loop system with some actuator time delays under white noise disturbance

time domain constraint is guaranteed to be less than its prescribed limit in spite of the large bump energy by the designed output feedback controller. It can be seen that

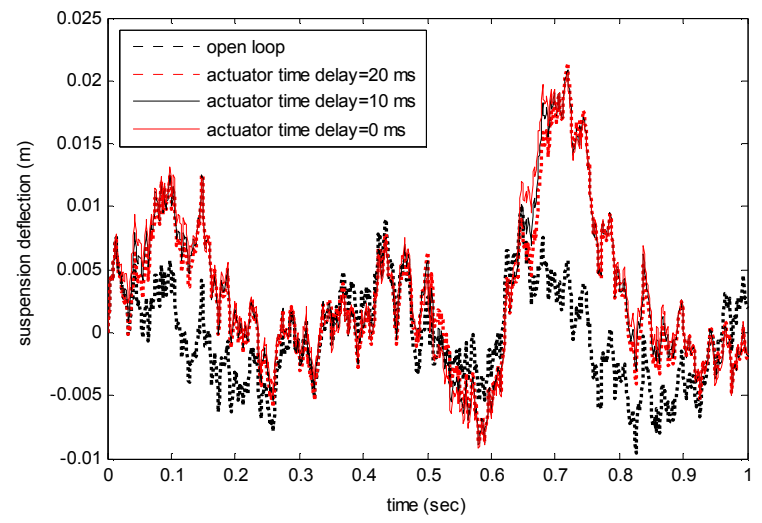

Fig. 13: Suspension deflections of open-loop system and closed-loop system with some actuator time delays under white noise disturbance

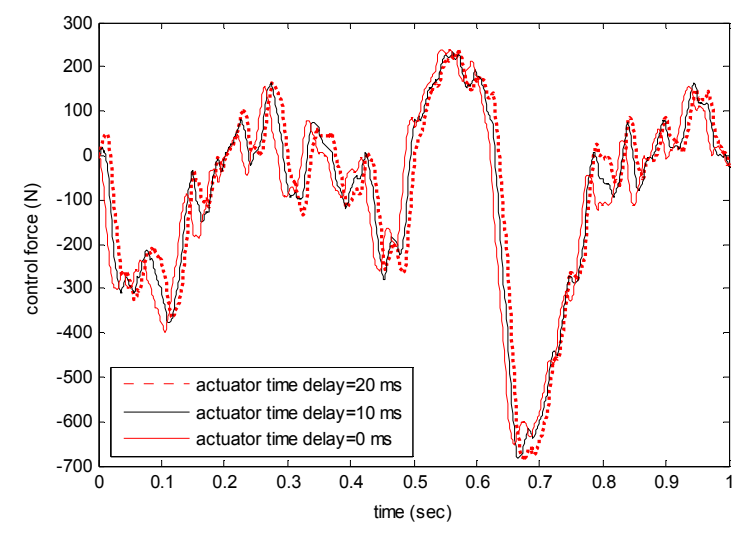

Fig. 14: Control forces of closed-loop system with some actuator time delays under white noise disturbance

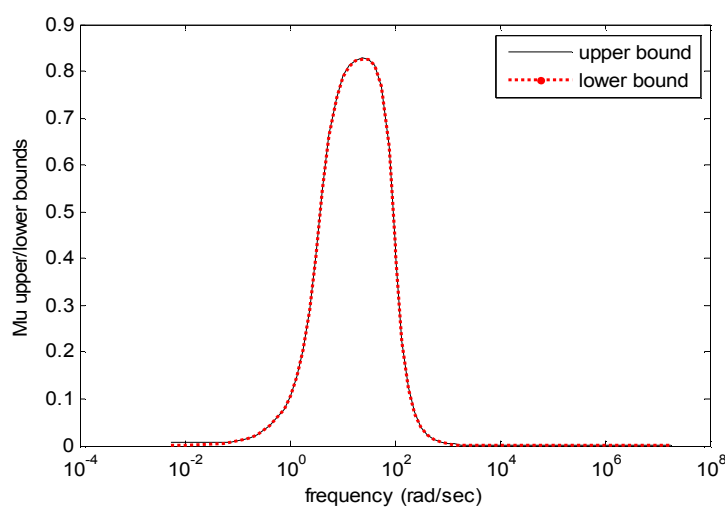

Fig. 15: $\mu$ lower/upper bounds of the uncertain closed-loop system

active controller increases the suspension deflection and actuator time delay has not a significant effect on the maximum value of the active suspension deflection.

Figure 11 depicts the active control forces of the closed-loop system, which are confined within a reasonable range and can be generated by hydraulic or electrorheological actuators in practice. It is confirmed 
that the designed robust active seat suspension system is able to guarantee a better performance under a pronounced bump disturbance and limited actuator control force in spite of the parameter uncertainty and uncertain actuator time delay.

Next, vertical human body accelerations of open and closed loop systems under white-noise input disturbance are illustrated in Fig. 12, from which it can be seen that the closed-loop system has a better performance with or without actuator time delays. However, the magnitude of the actuator time delay has not a significant effect on the controller performance in a large range of disturbance area.

The white noise disturbance response of suspension deflection is plotted in Fig. 13, from which it can be seen that this time domain constraint is guaranteed to be less than its prescribed limit.

The active control forces of the closed-loop system are depicted in Fig. 14, which are confined within the considered saturation range.

As one can see in this figure, the actuator time delay has not a meaningful effect on the controller performance, suspension deflection and actuator force under a random disturbance. In addition, closed-loop system performance under random disturbance is not as appropriate as its performance under bump disturbance and it is because of using $\mathrm{H} \infty$ norm for performance in the controller designing procedure.

Finally, for investigating the robustness of the closed-loop system performance, the lower/upper singular value $(\mu)$ bounds of the uncertain closed-loop system is depicted in Fig. 15. As it can be seen in this figure, these bounds are limited under to one in a wide range of frequencies. So, the robust performance of the closed-loop system by using of the designed controller is guaranteed.

\section{CONCLUSION}

This study establishes robust output feedback optimal $\mathrm{H}_{\infty}$ control synthesis for a class of uncertain seat suspension systems with actuator saturation and an uncertain actuator time delay. A four DOF human body model has been added to the seat suspension system to obtain a good tradeoff between performance and accuracy as well as a better insight of the controller design. In addition, two measurable states, in a real system, have been considered as output feedback. The optimal $\mathrm{H}_{\infty}$ controller has been obtained by solving a $D-K$ iteration algorithm. Note that, for actuator saturation considering, the control force is added to the desired output vector. Finally, a design example has been given to demonstrate the effectiveness of the proposed controller design approach. This design example showed that the closed-loop system has an appropriate performance, especially under a bump disturbance. Moreover, the closed-loop system has robust performance in a wide frequency range.

\section{REFERENCES}

Choi, S. and Y. Han, 2007. Vibration control of electro rheological seat suspension with human-body model using sliding mode control. J. Sound Vib., 303(1-2): 391-404.

Chou, J.H., S.H. Chen and F.Z. Lee, 1998. Grey-fuzzy control for active suspension design. Int. J. Vehicle Des., 19(1): 65-77.

$\mathrm{Du}, \mathrm{H}$. and N. Zhang, 2007. $\mathrm{H}_{\infty}$ control of active vehicle suspensions with actuator time delay. J. Sound Vib., 301(1-2): 236-252.

Du, H., K.Y. Sze and J. Lam, 2005. Semi-active $\mathrm{H}_{\infty}$ control of vehicle suspension with magnetorheological dampers. J. Sound Vib., 283(3-5): 981996.

El Madany, M.M. and M.I. Al-Majed, 2001. Quadratic synthesis of active controls for a quarter-car model. J. Vib. Control, 7(8): 1237-1252.

Esmailzadeh, E. and H.D. Taghirad, 1998. Active vehicle suspensions with optimal state-feedback control. Int. J. Model. Simul., 18(3): 228-237.

Gao, H. and C. Wang, 2003. Delay-dependent robust $\mathrm{H}_{\infty}$ and $\mathrm{L}_{2}-\mathrm{L}_{\infty}$ filtering for a class of uncertain nonlinear time-delay systems. IEEE T. Autom. Control, 48(9): 1661-1666.

Gao, H., J. Lam and C. Wang, 2006. Multi-objective control of vehicle active suspension systems via load-dependent controllers. J. Sound Vibrat., 290(3): 654-675.

Guo, L.X. and L.P. Zhang, 2012. Robust $\mathrm{H}_{\infty}$ control of active vehicle suspension under non-stationary running. J. Sound Vib., 331(26): 5824-5837.

He, Y., M. Wu, J. She and G. Liu, 2004. Delaydependent robust stability criteria for uncertain neutral systems with mixed delays. Syst. Control Lett., 51(1): 57-65.

Kiriczi, S. and R. Kashani, 1991. Robust control of active car suspension with model uncertainty using $\mathrm{H}_{\infty}$ methods. Adv. Automot. Technol. ASME, 40: 375-390.

Li, H., H. Liu, S. Hand and C. Hilton, 2012. Multiobjective $\mathrm{H}_{\infty}$ controls for vehicle active suspension systems with random actuator delay. Int. J. Syst. Sci., 43(12): 2214-2227.

Liang, C. and C. Chiang, 2006. A study on biodynamic models of seated human subjects exposed to vertical vibration. Int. J. Ind. Ergonom., 36(10): 869-890.

Ma, M., H. Chen and X. Liu, 2012. Robust H-infinity control for constrained uncertain systems and its application to active suspension. J. Control Theory Appl., 10(4): 470-476.

Meng, F.W., Z. He, Y. Wang and D. Zhou, 2009. $\mathrm{H}_{\infty}$ state feedback design of an electromagnetic suspension system. Dianji yu Kongzhi Xuebao/Electr. Mach. Control, 13(2): 282-286. 
Park, P., 1999. A delay-dependent stability criterion for systems with uncertain time-invariant delays. IEEE T. Autom. Control, 44(4): 876-877.

Ray, L.R., 1991. Robust linear-optimal control laws for active suspension systems. Adv. Automot. Technol. ASME, 40: 291-302.

Roh, H.S. and Y. Park, 1999. Stochastic optimal preview control of an active vehicle suspension. J. Sound Vib., 220(2): 313-330.

Salman, M.A., A.Y. Lee and N.M. Boustany, 1990. Reduced order design of active suspension control. J. Dyn. Syst. Meas. Control, 112(4): 604-610.

Shi, P., E. Boukas and R. Agarwal, 1999. Control of Markovian jump discrete-time systems with norm bounded uncertainty and unknown delay. IEEE T. Autom. Control, 44(11): 2139-2144.

Wang, Z., Y. Liu and X. Liu, 2005. On global asymptotic stability of neural networks with discrete and distributed delays. Phys. Lett. A, 345(4-6): 299-308.

Wang, Z., F. Yang, D. Ho and X. Liu, 2006. Robust $\mathrm{H}_{\infty}$ filtering for stochastic time-delay systems with missing measurements. IEEE T. Signal Process., 54(7): 2579-2587.
Watanabe, Y. and R.S. Sharp, 1999. Neural network learning control of automotive active suspension systems. Int. J. Vehicle Des., 21(2-3): 124-147.

Wei, L. and J. Griffin, 1998. The prediction of seat transmissibility from measures of seat impedance. J. Sound Vib., 214(1): 121-137.

Wu, L., P. Shi, C. Wang and H. Gao, 2006. Delaydependent robust $\mathrm{H}_{\infty}$ and $\mathrm{L}_{2}-\mathrm{L}_{\infty}$ filtering for LPV systems with both discrete and distributed delays. IEE P-Contr. Theor. Ap., 153(4): 483-492.

Yamashita, M., K. Fujimori, K. Hayakawa and H. Kimura, 1994. Application of $\mathrm{H}_{\infty}$ control to active suspension systems. Automatica, 30(11): 1717-1729.

Yoshimura, T., N. Ananthanarayana and D. Deepak, 1986. An active vertical suspension for track vehicle systems. J. Sound Vib., 106(2): 217-225.

Zhao, Y., W. Sun and H. Gao, 2010. Robust control synthesis for seat suspension systems with actuator saturation and time-varying input delay. J. Sound Vib., 329(21): 4335-4353. 